\title{
Molecular Microbiology in Antibacterial Research
}

\author{
You-Hee Cho* \\ Department of Pharmacy, College of Pharmacy, CHA University, \\ Gyeonggi-do 463-840, Republic of Korea
}

The special issue of Journal of Microbiology contains six reviews dealing with cutting edge research achievements in the fields of molecular microbiology focusing on antibacterial research. In a more specific sense, this special issue helps outline the progress of $21^{\text {st }}$-century basic molecular microbiology that can encompass related disciplines regarding a variety of interactions involving bacteria during bacterial pathogenesis and their control: sociomicrobiology (interaction between bacteria), immunology (interaction between bacteria and their hosts), and bacteriophage (phage) virology (interaction between bacteria and their parasites). Recent advancements have rapidly been made in our understanding of the real situation regarding polymicrobial interactions during bacterial infection and in non-mammalian host infection models to uncover the molecular mechanisms of host-bacteria interactions, which will complement our growing knowledge about immune responses toward bacterial and environmental elicitors. Moreover, much attention has recently been paid to phages and phage products as potential antibacterial therapeutics in the era of antibiotic resistance. Below, I summarize the individual contributions in these distinct categories.

\section{Polymicrobial synergy during infections: they are not alone}

The new knowledge in molecular microbiology is based on the multicellularity of microorganisms in a complex polymicrobial community: the cells in a microbial population might be heterogeneous in organized multicellular structures, where they undergo multicellular behaviors and interact with other cells in the same and other populations, constituting microbial communities. The totality of such microbes and their elements are defined as the microbiome, the constituents of which affect human physiology when they occur in the body. More importantly, human microbiome constituents can vary, resulting in the perturbation of the microbial balance, which can be caused by external and/or internal changes associated with infection, inflammation and so on.

${ }^{*}$ For correspondence. E-mail: youhee@cha.ac.kr; Tel.: +82-31-8017-9463; Fax: +82-31-8017-9420
Thus, it is quite natural to realize that all infectious diseases can actually be attributed to the outproliferation of harmful microbes over beneficial ones, whether or not they belong to the normal microbiome, and thus to the outcome of the microbial imbalance in infection sites of the human body. More importantly, not all microbial imbalances in the human body can be attributed to changes in a single microbial species, although the perturbation could be initiated by one species. Furthermore, the pathophysiological consequences of experimental polymicrobial infections are often worse than similar monomicrobial infections, suggesting potential synergistic interactions between harmful microbes during microbial imbalances. The review by Murray et al. (2014) focuses on polymicrobial synergy by describing the up-todate advances in the development of in vivo models and methods to analyze polymicrobial interactions.

\section{Non-mammalian host infections: a historical milestone in host-pathogen interaction}

Ever since the historical Irvine colloquium dedicated to the common virulence and defense mechanisms in host-pathogen interactions in plants and animals took place in 1999 (Keen et al., 2000), many microbiologists have been concentrating on developing appropriate non-mammalian model hosts to study microbial pathogenesis. Over the past decade, various model hosts have been chosen based on a number of traits that meet the need between similarity to mammals and genetic tractability, which include Arabidopsis thalinana, Caenorhabditis elegans, Drosophila melanogaster and Danio rerio. Among them, C. elegans has been extensively used to study host-pathogen interactions, leading to an innovative approach of a live-animal infection screen for new antimicrobials against Enterococcus faecalis, one of the ESKAPE pathogens notorious for antibiotic resistance. This in vivo screen enables us to identify new-paradigm antiinfectives that include antibiotics, antipathogenics and immune activators (Moy et al., 2006). Beside these achievements, more and more studies will be exploiting bacterial and host genetics to assess the molecular basis of the interface between hosts and bacteria, i.e. immunity and pathogenicity, based on the growing information on C. elegans immune responses at the molecular level. A comprehensive summary to inspire new prospects in host-pathogen interactions between enteroccoci and C. elegans is presented by Yuen and Ausubel (2014), one of the pioneering groups in multihost pathogenesis studies. 
Immunity and pathogenicity: new players in old diseases

Two reviews belonging to this category focus more on the health issues of progressive disorders in pulmonary functions. Chronic obstructive pulmonary disease (COPD) is an old lung disease characterized by chronically impaired airflow due to spontaneous tissue remodeling at the respiratory tract as aging progresses. COPD is currently the third major cause of death in the USA and more and more people will suffer from this disease in civilized environments, because the predominant etiology is exposure to excessive cigarette smoke and air pollutants that can compromise pulmonary immune functions. Many studies have been done to combat this illness mainly by understanding the pathological, physiological, and/or immunological aspects of COPD. In a broader sense, this pulmonary dysfunction can be considered as another cause of microbial imbalance in the upper and possibly lower respiratory tracts, and thus should also be viewed from a microbiological standpoint. All these aspects are summarized in the review by Hassett et al. (2014), with an emphasis on the microbiological perspective featuring Pseudomonas aeruginosa, the most common bacterial species isolated from COPD patients, which can prosper in the limited oxygen supply via anaerobiosis within its complex biofilm community.

The second review by Walling and Lau (2014) describes new in vitro and in vivo studies to understand pulmonary dysfunction upon exposure to air pollutants that provoke similar responses observed in COPD. Among many ultrafine particles that have been exploited for use as experimental air pollutants, nanoparticles and, more importantly, carbon nanotubes (CNTs), are noteworthy in that they have been incorporated in more and more industrial products nowadays. The altered immune functions and pathogen susceptibilities mediated by nanoparticles and CNTs are under active investigation using the animal models to decipher the details of the intimate, inevitable, and complex interactions in the respiratory tracts which involves the host, environmental, and microbial factors.

\section{Phage biology and beyond: friends or foes?}

The last important player in this issue is the bacterial viruses, i.e. phages that infect and normally kill their bacterial prey. Since their discovery back in the early 1900s, phages have been viewed not only as important genetic tools but also as potential antibacterial modalities, especially in the era of antibiotic resistance. They are the simplest organism to study, which gave birth to molecular biology; they are the simplest organism to evolve and diversify themselves to possess an enormous arsenal of genetic resources; and they are the simplest organism to engineer to be new artificial entities that can be tailored for human exploitation. The premise that phages have intelligently evolved multiple strategies to interfere with bacterial physiology enables us to study and exploit them and their gene products for new-concept antibacterial research. In this regard, two reviews are presented that cover phages and phage products: the one by Bondy-
Denomy and Davidson (2014) focuses on lysogeny-mediated changes in bacterial physiology, whereas the other by Young (2014) describes the molecular mechanisms of bacterial cell lysis caused by phage proteins. Once a temperate phage lysogenizes its bacterial host cell, it can affect the physiological traits of the bacterial lysogen, which can be regarded as a symbiotic state: superinfection exclusion, virulence, motility, antibiotic resistance and other traits can be affected by the balance between prophage activity and bacterial immunity, in part represented by the CRISPR (clustered regularly interspaced short palindromic repeats)-mediated adaptive immune system. Old phage proteins with muralytic activity to hydrolyze peptidoglycans have been shown to successfully eliminate bacterial pathogens upon direct administration into infected animals (Schuch et al., 2002). These phage proteins are endolysins that are normally synthesized inside cells and thus should be translocated by the holin complex. In contrast, some other endolysins with SAR (signal anchor release) sequences are normally localized in the periplasm, but remain inactive until the pinholin complex depolarizes the membrane. These two double-player pathways require a third player called spannins that can disrupt the outer membranes of Gram-negative bacteria to release progeny phages. The molecular details are well documented based on the chronological lineup of the relevant studies, helping us to gain insight into the molecular engineering of these useful enzymes.

I very much hope that the expert scientific knowledge regarding these concise molecular microbiology aspects presented in distinct but complementary categories will stimulate antibacterial research on a fundamental basis to further enable us to understand and manipulate bacterial natures. I am grateful to the authors for willingly providing their precious endeavors and the editorial board for providing the opportunity to orchestrate this special issue. The content of this special issue will surely attract the attention of the audience in our community.

\section{References}

Bondy-Denomy, J. and Davidson, A.R. 2014. When a virus is not a parasite: the beneficial effects of prophages on bacterial fitness. J. Microbiol. 52, 235-242.

Hassett, D.J., Borchers, M.T., and Panos, R.J. 2014. Chronic obstructive pulmonary disease (COPD): evaluation from clinical, immunological and bacterial pathogenesis perspectives. J. Microbiol. 52, 211-226.

Keen, N.T., Staskawicz, B.J., Mekalanos, J.J., Ausubel, F.M., and Cook, R.J. 2000. Pathogens and hosts: The dance is the same, the couples are different. Proc. Natl. Acad. Sci. USA 97, 87528753.

Moy, T.I., Ball, A.R., Anklesaria, Z., Casadei, G., Lewis, K., and Ausubel, F.M. 2006. Identification of novel antimicrobials using a live-animal infection model. Proc Natl. Acad. Sci. USA 103, 10414-10419.

Murray, J.L., Connell, J.L., Stacy, A., Turner, K.H., and Whiteley, M. 2014. Mechanisms of synergy in polymicrobial infections. J. Microbiol. 52, 188-199.

Schuch, R., Nelson, D., and Fischetti, V.A. 2002. A bacteriolytic agent 
that detects and kills Bacillus anthracis. Nature 418, 884-889.

Walling, B.E. and Lau, G.W. 2014. Perturbation of pulmonary immune functions by carbon nanotubes and susceptibility to microbial infection. J. Microbiol. 52, 227-234.

Young, R. 2014. Phage lysis: three steps, three choices, one outcome.
J. Microbiol. 243-258.

Yuen, G.J. and Ausubel, F.M. 2014. Enterococcus infection biology: lessons from invertebrate host models. J. Microbiol. 52, 200210 . 